\title{
Odontology prize 2017
}

Yuichi Tamaki, D.D.S., Ph.D., Department of Developmental and Regenerative Dentistry, The Nippon Dental University School of Life Dentistry at Tokyo, JAPAN, was awarded the ODONTOLOGY PRIZE 2017 for the best science paper in recognition of outstanding originality and significance in the paper.
"In vitro analysis of mesenchymal stem cells derived from human teeth and bone marrow".

Odontology, volume 101, number 2, page 121-32, 2013 July. 